\title{
Aplicación móvil en el diagnóstico de dolor de hombro para médicos generales
}

\section{Mobile Application in the Diagnosis of Shoulder Pain for General Physicians}

\author{
Michael Marsalli ${ }^{1,2}$ Oscar Sepúlveda ${ }^{3}$ Joaquín De La Paz ${ }^{3}$ \\ ${ }^{1}$ Equipo de Hombro, Departamento Traumatología y Ortopedia, \\ Hospital del Trabajador, Santiago, Chile \\ ${ }^{2}$ Equipo de Hombro Clínica Universidad de los Andes, Chile

\begin{abstract}
Address for correspondence Michael Marsalli, Equipo de Hombro, Departamento Traumatología y Ortopedia, Hospital del Trabajador, (e-mail: mmarsalli@hts.cl).
\end{abstract}

3 Becario programa Ortopedia y Traumatología, Universidad Andrés Bello, Chile

Rev Chil Ortop Traumatol 2020;61:47-52.

\section{Resumen}

Palabras claves

- dolor de hombro

- aplicación móvil

- diagnóstico dolor de hombro

Abstract
Objetivo Evaluar la concordancia diagnóstica durante la evaluación inicial de pacientes con dolor de hombro entre: (1) Traumatólogos especialistas en hombro realizando una evaluación convencional y (2) un médico general utilizando una nueva aplicación móvil.

Materiales y Método Se diseñó una aplicación móvil para asistir a médicos generales a realizar una evaluación del dolor de hombro entregando una hipótesis diagnóstica. Se diseñó un estudio de concordancia diagnóstica. Se evaluó un grupo de 60 pacientes, quienes consultaron por primera vez con un especialista en hombro. Primero, todos fueron evaluados por un médico general utilizando la aplicación. Se registró la hipótesis diagnóstica entregada por la aplicación. Luego, el especialista realizó una evaluación con anamnesis y examen físico, registrando su hipótesis diagnóstica. Se realizó una prueba Kappa para determinar la concordancia entre ambos evaluadores.

Resultados La concordancia global entre los especialistas y la aplicación móvil utilizada por el médico general fue sustancial $(\mathrm{k}=0,74 ; p<0,001)$. La concordancia fue casi perfecta para el Pinzamiento subacromial, dolor cervical, lesión acromioclavicular, inestabilidad de hombro, y el pinzamiento interno.

Conclusión La concordancia diagnóstica durante la evaluación inicial de pacientes con dolor de hombro entre traumatólogos especialistas y un médico general utilizando una aplicación móvil fue sustancial.

Nivel de Evidencia Tipo III.

Objective To evaluate the diagnostic concordance during the initial evaluation of patients with shoulder pain between: (1) Shoulder orthopedic surgeons performing a conventional evaluation and (2) a general practitioner using a new mobile application. Materials and Method A mobile application was designed to assist general practitioners to perform an evaluation of shoulder pain by providing a diagnostic hypothesis. A diagnostic concordance study was designed. A group of 60 patients were received

July 18, 2019

accepted

May 13, 2020
DOI https://doi.org/

10.1055/s-0040-1713797. ISSN 0716-4548.
Copyright $\odot 2020$ by Thieme Revinter

Publicações Ltda, Rio de Janeiro, Brazil
License terms

()ㅇㅇ $\Theta \circledast$ 


\section{Keywords}

- shoulder pain

- mobile application

- shoulder pain diagnosis evaluated, who consulted for the first time with a shoulder specialist. First, they were all evaluated by a general practitioner using the application. The diagnostic hypothesis delivered by the application was recorded. Then, the specialist made an evaluation with anamnesis and physical examination, recording his diagnostic hypothesis. A Kappa test was performed to determine the agreement between both evaluators.

Results The overall agreement between the specialists and the mobile application used by the general practitioner was substantial $(\mathrm{k}=0.74, p<0.001)$. The agreement was almost perfect for subacromial impingement, cervical pain, acromioclavicular injuries, shoulder instability, and internal impingement.

Conclusion The diagnostic agreement during the initial evaluation of patients with shoulder pain between shoulder specialist and a general practitioner using a mobile application was substantial.

Level of Evidence Type III.

\section{Introducción}

Las aplicaciones médicas móviles han tenido un auge importante durante los últimos años. Es posible encontrar muchas de ellas de acceso gratuito desde cualquier dispositivo móvil. Tal ha sido su impacto, que la Organización Mundial de la Salud (OMS), ha acuñado el término de Mobile Health (mHealth) para referirse a todas las prácticas médicas y de salud pública soportadas por dispositivos móviles. ${ }^{1,2}$ De la misma manera, la agencia "Food and Drug Administration" de los Estados Unidos, ha generado guías para regularlas. El año 2013 emitió una guía (actualizada posteriormente en el 2015), para aplicaciones médicas móviles que apoya la innovación y protege la salud de los pacientes. ${ }^{3}$

El "dolor de hombro" puede ser causado por una amplia gama de patologías propias del hombro o bien ser un dolor referido. ${ }^{4}$ Es un cuadro común que representa la tercera causa de consulta por trastornos musculoesqueléticos en la atención primaria. ${ }^{5}$ El enfrentamiento del dolor de hombro en la atención primaria supone un desafío al médico no especialista, debido a la gran cantidad de diagnósticos diferenciales. En ese escenario, resulta relevante realizar una aproximación diagnóstica correcta en la primera evaluación para indicar el estudio necesario y derivar al especialista solo cuando corresponda. Así mismo, evitar derivaciones y exámenes innecesarios que pudieran causar tiempos prolongados de atención y costos adicionales.

Dado lo frecuente del dolor de hombro como motivo de consulta en la atención primaria, surge la necesidad de evaluar herramientas que faciliten el proceso diagnóstico al médico no especialista. ${ }^{6}$ Los avances de la tecnología móvil representan una oportunidad para diseñar y evaluar herramientas digitales que faciliten el diagnóstico inicial de un paciente con dolor de hombro. Sin embargo, esas aplicaciones deben demostrar su utilidad clínica.

Previo a este estudio, se diseñó una aplicación móvil con el objetivo de asistir a médicos no entrenados a realizar una aproximación diagnóstica de pacientes con dolor de hombro. Su función es obtener una hipótesis diagnóstica que sea similar a la de una evaluación por un especialista en hombro.
El objetivo principal de este estudio fue evaluar la concordancia diagnóstica durante la evaluación inicial de pacientes con dolor de hombro entre: Traumatólogos especialistas en hombro realizando una evaluación convencional y un médico general utilizando una aplicación móvil diseñada para esos fines.

La hipótesis de este estudio fue que los diagnósticos planteados entre la evaluación del grupo de traumatólogos especialistas en hombro y el médico general usando la aplicación móvil sería similar.

\section{Materiales y Método}

\section{Desarrollo de la Aplicación Móvil}

Previo al inicio del estudio, se diseñó una aplicación móvil basada en una metodología de anamnesis guiada según las respuestas del paciente. Los objetivos del diseño de la aplicación fueron los siguientes:

1. Entregar una hipótesis diagnóstica con respecto al dolor de hombro en el escenario de una consulta ambulatoria y electiva.

2. Ser utilizado por personal de salud no capacitado en la evaluación específica del dolor de hombro.

3. Que fuese una guía para las conductas clínicas y los estudios complementarios.

Para ello, se revisaron otras herramientas digitales con objetivos similares encontradas en internet que pudiesen servir como referencia. Se consideraron las herramientas "Symptom Checker" del sitio web de Clínica Mayo, "Self diagnosis symptom checker" del sitio web de Clínica Stone, "Shoulder pain" del sitio web de Cleveland Clinic y "Diagnose Your Shoulder" del sitio web ShoulderDoc. ${ }^{7-10}$

Luego, se redactó un flujograma de preguntas y respuestas que terminaron en 10 posibles resultados. Nueve resultados fueron diagnósticos diferenciales de dolor de hombro más su estudio sugerido y una décima opción que fue "No se logró realizar el diagnóstico.” Esas 9 hipótesis diagnósticas fueron: Pinzamiento subacromial, rotura del manguito rotador, capsulitis adhesiva, lesión traumática acromioclavicular, 
artrosis de hombro, artrosis acromioclavicular, inestabilidad de hombro, SLAP y/o pinzamiento interno y por último Sd. Dolor Cervical.

Posteriormente, se eligió a la agencia digital Reactor ${ }^{\circledR}$ Spa. para trabajar en conjunto el diseño y la programación de la aplicación digital. ${ }^{11}$ Como resultado se obtuvo la primera versión de esta aplicación digital, la cual se diseñó para que respondiera a pantallas de escritorio y pantallas móviles de todos los tamaños (- Figura $\mathbf{1}$ ).

\section{Diseño del Estudio}

Se diseñó un estudio de concordancia diagnóstica. Se calculó un tamaño de la muestra para un estadígrafo kappa esperado de 0,75 con el programa EPIDAT ${ }^{\circledR}$ (versión 4.1), obteniéndose un valor mínimo de 43 pacientes.

Se incluyeron pacientes mayores de 18 años, que consultaron de forma electiva por dolor de hombro y por primera vez con algún traumatólogo especialista en hombro en un único centro médico.

Se excluyeron pacientes en situación de discapacidad mental temporal o permanente y pacientes con diagnóstico de fracturas u otra condición que requiera atención de urgencia.

Los pacientes fueron invitados a participar del estudio al momento de presentarse en el centro de especialidades médicas. Se les explicó detalladamente los objetivos y el método del trabajo. Antes de ingresar a la atención con el traumatólogo especialista en hombro, un médico general evaluó a los pacientes utilizando exclusivamente el flujograma de preguntas de la aplicación móvil. Esa evaluación por el médico general, no incluyó examen físico. El médico dispuso de un dispositivo móvil con conexión a internet con la aplicación lista para ser utilizada.

Al obtener una hipótesis diagnóstica desde la aplicación móvil, ella quedó registrada por el médico general. Posteriormente, el mismo paciente fue evaluado por el especialista en hombro que le correspondía, quien se mantuvo ciego al resultado obtenido por el médico general utilizando la aplicación. El especialista realizó su anamnesis junto con un examen físico al paciente y registró su hipótesis diagnóstica. Eso fue realizado antes de revisar cualquier tipo de examen en caso de que el paciente los hubiese tenido durante su primera consulta con el especialista en hombro. Participaron un total de 5 traumatólogos especialistas en hombro en la evaluación de los pacientes incluidos. Todos ellos con al menos 3 años de experiencia como subespecialistas en hombro.

La recolección de la información se realizó de manera transversal. Las hipótesis diagnósticas fueron tabuladas como base de datos encriptadas. Los resultados de concordancia diagnóstica entre la aplicación móvil y los especialistas en hombro se presentaron calculando el índice de kappa (- Tabla 1). ${ }^{12}$ Esos cálculos fueron realizados en el programa estadístico IBM SPSS Statistics Base (versión 22.0). Se consideró un valor estadísticamente significativo para un valor $\mathrm{p} \leq 0,05$.

Se solicitó la evaluación y aprobación del comité de ética hospitalario, en cuya composición no participa ninguno de los autores de la investigación. Todos los pacientes incluidos firmaron un consentimiento informado previo al ingreso al estudio.

\section{Resultados}

\section{Número de Pacientes}

Se incluyeron 60 pacientes consecutivos que consultaron por primera vez por dolor de hombro en el centro de

Tabla 1 Índice de Kappa y fuerza de concordancia

\begin{tabular}{|l|l|}
\hline Kappa & Fuerza de la concordancia \\
\hline$<0$ & Pobre \\
\hline $0-0,2$ & Leve \\
\hline $0,21-0,4$ & Aceptable \\
\hline $0,41-0,6$ & Moderada \\
\hline $0,61-0,8$ & Sustancial \\
\hline $0,81-1$ & Casi Perfecta \\
\hline
\end{tabular}

\begin{tabular}{|c|c|c|c|}
\hline 同 & กิ & 同 & ก \\
\hline Diagnóstico dolor de hombro & Diagnóstico dolor de hombro & Diagnóstico dolor de hombro & Diagnóstico dolor de hombro \\
\hline $\begin{array}{l}\text { ¿En qué zona se encuentra el } \\
\text { dolor? }\end{array}$ & $\begin{array}{l}\text { ¿Cuándo presentas mayor dolor? } \\
\text { Al levantar el brazo sobre la cabeza } \\
\text { Al bajar el brazo desde la altura } \\
\text { Las dos alternativas anteriores } \\
\text { No puedo levantar el brazo } \\
\text { Ninguna de las anteriores }\end{array}$ & $\begin{array}{l}\text { No puedo levantar el brazo } \\
\text { ¿Está déblil? Puedo levantar e brazo con la ayuda } \\
\text { de la otra mano } \\
\text { EEstá ríidio? No puedo levantar el brazo aunque lo } \\
\text { intente con la ayuda de la otra mano }\end{array}$ & 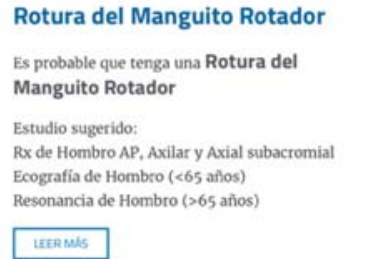 \\
\hline 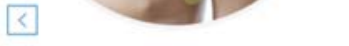 & 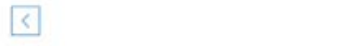 & $\square$ & $<$ \\
\hline
\end{tabular}

- Las imágenes son solo un ejemplo y no se presentan de manera ordenada o secuencial

Fig. 1 Imágenes referenciales* de la aplicación móvil para la evaluación y diagnóstico del dolor de hombro. 
especialidades médicas con un especialista. Catorce (23\%) de los pacientes, fueron excluidos del análisis de concordancia por presentar un diagnóstico que solo se encontró en los resultados de la aplicación móvil o solo en el del especialista. Eso se debe a que se requiere que un diagnóstico se encuentre al menos una vez en cada lado para el análisis de concordancia. Se incluyeron un total de 46 pacientes para el análisis de concordancia diagnóstica.

\section{Capacidad Diagnóstica}

Los especialistas del equipo de hombro plantearon una hipótesis diagnóstica en el $100 \%$ de los casos evaluados mediante la anamnesis y el examen físico. El médico general, con solo el uso de la aplicación móvil fue capaz de plantear una hipótesis diagnóstica en el 88\% (53) de los casos evaluados. En 7 casos, el resultado de la aplicación fue "No hemos sido capaces de realizar un diagnóstico."

\section{Concordancia Diagnóstica}

La concordancia global entre los especialistas en hombro y la aplicación móvil utilizada por el médico general fue sustancial $(\mathrm{k}=0,74 ; p<0,001)$. La concordancia evaluada de manera individual fue casi perfecta $(\mathrm{k}=>0,8)$ para el Sd. pinzamiento subacromial, Sd. Dolor cervical, lesión traumática acromioclavicular, inestabilidad de hombro, y el Sd. Pinzamiento interno/lesión tipo SLAP. El diagnóstico de rotura del manguito rotador tuvo una concordancia diagnóstica de $\mathrm{k}=0,79$ (sustancial). En esa serie, la aplicación móvil no presentó como resultados los diagnósticos de capsulitis adhesiva ni artrosis de hombro. El especialista no planteó los diagnósticos de artrosis acromioclavicular ni artrosis de hombro (-Tabla 2).

Tabla 2 Concordancia y frecuencia para los diferentes diagnósticos entre la evaluación del médico general con el uso de la aplicación móvil y el traumatólogo especialista en hombro

\begin{tabular}{|l|l|l|l|l|}
\hline Diagnóstico & APP & TMT & $\begin{array}{l}\text { Concordancia } \\
\text { (Kappa) }\end{array}$ & Valor p \\
\hline Global & 46 & 46 & 0,74 & $<0.001$ \\
\hline $\begin{array}{l}\text { Rotura del } \\
\text { manguito } \\
\text { rotador }\end{array}$ & 28 & 35 & 0,79 & $<0.001$ \\
\hline $\begin{array}{l}\text { Sd. Pinzamiento } \\
\text { Subacromial }\end{array}$ & 8 & 6 & 0,83 & $<0.001$ \\
\hline Sd. Dolor Cervical & 2 & 1 & 0,91 & $<0.001$ \\
\hline $\begin{array}{l}\text { Lesión traumática } \\
\text { acromioclavicular }\end{array}$ & 2 & 1 & 0,95 & $<0.001$ \\
\hline $\begin{array}{l}\text { Inestabilidad } \\
\text { de hombro }\end{array}$ & 2 & 1 & 0,95 & $<0.001$ \\
\hline $\begin{array}{l}\text { Sd. Pinzamiento } \\
\text { interno/SLAP }\end{array}$ & 2 & 1 & 0,98 & $<0.001$ \\
\hline $\begin{array}{l}\text { Artrosis } \\
\text { acromioclavicular }\end{array}$ & 2 & 0 & - & \\
\hline Capsulitis adhesiva & 0 & 1 & - & \\
\hline Artrosis de hombro & 0 & 0 & - & \\
\hline
\end{tabular}

Tabla 3 Diagnósticos excluidos del análisis de concordancia

\begin{tabular}{|l|l|}
\hline Del Traumatólogo & Número de pacientes \\
\hline Tenosinovitis bicipital & 2 \\
\hline Contusión de hombro & 3 \\
\hline Fibromialgia & 1 \\
\hline Lesión tumoral del hombro & 1 \\
\hline De la Aplicación & 7 \\
\hline $\begin{array}{l}\text { "No hemos sido capaces } \\
\text { de realizar un diagnóstico" }\end{array}$ & \\
\hline
\end{tabular}

\section{Diagnósticos Excluidos del Análisis de Concordancia}

Se excluyeron del análisis Kappa de concordancia 4 diagnósticos en un total de $7(11,6 \%)$ pacientes que fueron planteados por los especialistas y que no estaban dentro de las opciones programadas en la aplicación. Se excluyeron también los 7 pacientes $(11,6 \%)$ en donde la aplicación planteó la opción "No hemos sido capaces de realizar un diagnóstico" lo cual no fue planteado en ningún caso por los especialistas ( - Tabla 3 ).

\section{Discusión}

Con el uso de esa aplicación, el médico general fue capaz de realizar una hipótesis diagnóstica de trabajo en el $88 \%$ de los pacientes evaluados. Eso se asoció a una concordancia global sustancial con los especialistas en hombro que participaron en este estudio. La alta concordancia global, permite pensar que la evaluación médica con esa aplicación planteará una hipótesis diagnóstica similar al de un especialista en hombro en la mayoría de los pacientes en el contexto de la primera evaluación, más allá del azar.

Sin embargo, se debe considerar la baja incidencia de algunos diagnósticos en esa serie. La mayoría de los diagnósticos no relacionados con patología alrededor del manguito rotador fue diagnosticado en 2 o menos pacientes por uno de los evaluadores. Un índice de kappa específico alto en esos casos, se explica más bien por la alta tasa que existió al no diagnosticar esa enfermedad en particular y a su vez, lograr acuerdo en la presencia de otra, siendo la distribución en ambos evaluadores bastante similar. Cuántas más categorías se estén considerando, más difícil será clasificar correctamente los sujetos estudiados, lo que habitualmente implica valores de kappa más bajos.

De todas formas, es recomendable en una siguiente versión, aumentar el número total de pacientes estudiados para determinar si la concordancia se mantiene con una mayor incidencia de casos con diagnósticos de baja frecuencia. Así, podremos observar si los acuerdos se mantienen en casos positivos para esas patologías, tanto como en los casos negativos.

Los beneficios de ese tipo de tecnología son múltiples. Por una parte, se puede estandarizar la evaluación en el enfrentamiento inicial de pacientes con dolor de hombro. Segundo, un diagnóstico inicial similar al de un especialista, permite protocolizar las recomendaciones al médico general para tomar las conductas más adecuadas. Por ejemplo, 
indicar el estudio inicial correcto u orientar con criterios de derivación específicos. Tercero, los beneficios de esa tecnología aplicada a una red asistencial podrían evitar gastos de estudios innecesarios y disminuir los tiempos de espera para comenzar con los tratamientos adecuados.

En esta evaluación, mediante una anamnesis estandarizada asistida por esta aplicación, no se realizó un examen físico del hombro. Las diferentes pruebas del examen físico del hombro, han demostrado una alta variabilidad en el rendimiento para diagnósticos como el sd. pinzamiento subacromial, roturas del manguito rotador, lesiones del tendón del bíceps, artrosis acromioclavicular y lesiones labrales. ${ }^{13,14}$ Por lo tanto, una herramienta digital validada puede facilitar y complementar las evaluaciones clínicas realizadas por médicos no entrenados. Creemos que, en un futuro, se deben evaluar los resultados diagnósticos entre la aplicación asociada al examen físico del médico general contra la evaluación del especialista. De esa manera, podremos evaluar la contribución del examen físico a la evaluación del médico general y probablemente, mejorar la concordancia con el especialista. Por otro lado, apoyarse en una anamnesis estructurada con buen rendimiento, nos hace pensar en expandir su uso hacia el telediagnóstico y la telemedicina apoyando las evaluaciones iniciales.

Los especialistas no plantearon 2 de los 9 diagnósticos incluidos en la aplicación. Hubo 4 diagnósticos realizados por los especialistas en 7 pacientes, que no se presentaron como una alternativa incluida en la aplicación. Interpretamos que los diagnósticos incluidos en la aplicación, son acordes con los más frecuentes que se presentan como $\mathrm{Sd}$. de hombro doloroso en la consulta ambulatoria habitual, pero debemos aumentar el número de pacientes evaluados para realizar futuras modificaciones. Incluir más diagnósticos es una alternativa, pero se debe considerar que mientras tengamos más opciones, más riesgo habrá de tener una menor concordancia. Decidimos excluir los diagnósticos relacionados a fracturas, ya que en general, su diagnóstico es más evidente en el contexto de una consulta con antecedentes traumáticos agudos, impotencia funcional y a veces deformidad visible.

También, hubo 2 diagnósticos que, a pesar de haber sido planteados al menos una vez cada uno por los especialistas, la aplicación no fue capaz de plantearlos en toda esta serie. Esos diagnósticos deberán ser revisados evaluando más pacientes o bien mejorando las preguntas y respuestas para hacerlas más precisas.

No existen hasta la fecha, otras aplicaciones digitales para la evaluación del dolor de hombro estudiadas en la literatura. Tampoco encontramos otras aplicaciones para el apoyo diagnóstico en ortopedia. Se han estudiado aplicaciones con buenos resultados para otras especialidades y de maneras muy variadas. Por ejemplo, en el seguimiento a distancia de heridas para pacientes con poco acceso a atención médica, asistencia en el control del asma en adolecentes, recordatorios de citas médicas o estimación de los niveles de hemoglobina con la cámara del teléfono móvil, gran parte de ellos con buenos resultados. ${ }^{15-18}$ Frente a eso, durante el año 2018, la OMS publicó una clasificación numérica para las tecnologías digitales y móviles, basada en su usuario primario objetivo. ${ }^{19}$ La aplicación presentada en ese estudio es categorizada como 2.3, al ser de apoyo en las decisiones del proveedor de salud.

Las limitaciones de esa aplicación para la evaluación de pacientes con dolor de hombro son básicamente dos: 1) necesidad de contar con un dispositivo que tenga las características técnicas suficientes y acceso a red de internet y 2) la cantidad de diagnósticos que se decide incluir como alternativas de resultado.

La experiencia con esa primera versión permitió proponer las siguientes mejoras para una segunda versión. Ellas incluyeron: 1) Mejorar la precisión del flujo de preguntas y respuestas de los diagnósticos que no fueron planteados como resultados en esta serie de pacientes. 2) Mejorar el diseño aumentando el tamaño de la fuente. 3) Mejorar la usabilidad aumentando la discriminación entre 2 puntos para evitar errores al pulsar las alternativas de respuestas con el dedo pulgar.

En un futuro, esa aplicación debe ser reevaluada con esas mejoras, mayor número de pacientes y otros grupos de especialistas en hombro. Además, creemos que se debe evaluar su usabilidad y rendimiento en el contexto del telediagnóstico, en donde una anamnesis sistematizada asociada a algunas pruebas del examen físico que no requieren ser realizadas de manera presencial (inspección y rangos de movimientos), pudiesen tener una buena concordancia con una evaluación presencial de un especialista en hombro.

\section{Conclusiones}

La concordancia diagnóstica durante la evaluación inicial de pacientes con dolor de hombro entre traumatólogos especialistas en hombro realizando una evaluación convencional y un médico general utilizando una aplicación móvil diseñada para esos fines fue sustancial. La aplicación logró plantear una hipótesis diagnóstica en la mayoría de los pacientes que consultaron por dolor de hombro de manera electiva y ambulatoria. Sin embargo, se debe reevaluar la reproducibilidad de esos resultados con un mayor número de pacientes. En un futuro, se deben mejorar aspectos de su diseño y reevaluar los diagnósticos incluidos para lograr realizar una hipótesis diagnóstica en todos los pacientes y mejorar su concordancia con el especialista.

Conflictos de intereses

Ninguno

\section{Bibliografía}

1 WHO Global Observatory for eHealth. mHealth: new horizons for health through mobile technologies: second global survey on eHealth. Geneva: World Health Organization; 2011. [Internet]. Available from: http://www.who.int/iris/handle/10665/44607

2 Singh P, Panjwani M. mHealth: Meaning, Purposeand Outcomes. IJCSIT 2016;7(05):2216-2221

3 U.S. Department of Health and Human Services Food and Drug Administration. Mobile Medical Applicationes. Guidance for Industry and Food and Drug Administration Staff [Internet]. 2015. Available from: https://www.fda.gov/downloads/MedicalDevices/ DeviceRegulationandGuidance/GuidanceDocuments/UCM263366. pdf 
4 Greenberg DL. Evaluation and treatment of shoulder pain. Med Clin North Am 2014;98(03):487-504

5 Urwin M, Symmons D, Allison T, et al. Estimating the burden of musculoskeletal disorders in the community: the comparative prevalence of symptoms at different anatomical sites, and the relation to social deprivation. Ann Rheum Dis 1998;57(11):649-655

6 Tzortziou Brown V, Underwood M, Mohamed N, Westwood O, Morrissey D. Professional interventions for general practitioners on the management of musculoskeletal conditions. Cochrane Database Syst Rev 2016;(05):CD007495

7 Mayo Clinic. Symptom Checker [Internet]. Available from: https:// www.mayoclinic.org/symptom-checker/select-symptom/itt-2000 9075

8 The Stone Clinic. Self diagnosis symptom checker [Internet]. Available from: https://www.stoneclinic.com/shoulder-self-diagnosis

9 Cleveland Clinic. Shoulder Pain [Internet]. Available from: https://pages.clevelandclinic.org/shoulder-pain-index.html

10 ShoulderDoc. Diagnose Your Shoulder [Internet]. Available from: https://www.shoulderdoc.co.uk

11 Reactor Spa [Internet]. Available from: https://www.reactor.cl

12 Landis JR, Koch GG. The measurement of observer agreement for categorical data. Biometrics 1977;33(01):159-174

13 Hegedus EJ, Goode AP, Cook CE, et al. Which physical examination tests provide clinicians with the most value when examining the shoulder? Update of a systematic review with meta-analysis of individual tests. Br J Sports Med 2012;46(14):964-978

14 The Cochrane Collaboration, Ed. Cochrane Database of Systematic Reviews: Protocols [Internet]. Chichester, UK: John Wiley \& Sons, Ltd; 1996 [cited 2019 Feb 6]. Available from: http://doi.wiley. com/10.1002/14651858

15 Ye J, Zuo Y, Xie T, et al. A telemedicine wound care model using 4G with smart phones or smart glasses: A pilot study. Medicine (Baltimore) 2016;95(31):e4198

16 Alquran A, Lambert KA, Farouque A, et al. Smartphone Applications for Encouraging Asthma Self-Management in Adolescents: A Systematic Review. Int JEnviron Res Public Health 2018;15(11):2403

17 da Costa TM, Salomão PL, Martha AS, Pisa IT, Sigulem D. The impact of short message service text messages sent as appointment reminders to patients' cell phones at outpatient clinics in São Paulo, Brazil. Int J Med Inform 2010;79(01):65-70

18 Mannino RG, Myers DR, Tyburski EA, et al. Smartphone app for non-invasive detection of anemia using only patient-sourced photos. Nat Commun 2018;9(01):4924http://www.nature.com/ articles/s41467-018-07262-2 [Internet]

19 World Health Organization. Classification of Digital Health Interventions. 2018 [Internet]. Available from: https://www. who.int/reproductivehealth/publications/mhealth/ classification-digital-health-interventions/en/ 